\title{
Direct numerical simulations on crack formation in ceramic materials under thermal shock by using a non-local fracture model
}

\author{
$\mathrm{Jia} \mathrm{Li}^{\mathrm{a}, *}$, Fan Song ${ }^{\mathrm{b}}$, Chiping Jiang ${ }^{\mathrm{c}}$ \\ a LSPM, CNRS UPR 3407, Université Paris XIII, Villetaneuse, France \\ b State Key Laboratory of Nonlinear Mechanics, Institute of Mechanics, Chinese Academy of Sciences, Beijing, China \\ c Solid Mechanics Research Center, Beijing University of Aeronautics and Astronautics, Beijing, China
}

Received 11 November 2012; received in revised form 15 April 2013; accepted 16 April 2013

Available online 14 May 2013

\begin{abstract}
In this work, a non-local failure model was proposed and implemented into a finite element code. It was then used to simulate the crack evolution in ceramic materials subjected to thermal shock. By using this numerical model, the initiation and propagation of cracks in water quenched ceramic specimens were simulated. The numerical simulations reproduced faithfully the crack patterns in ceramic specimens underwent quenching tests. The periodical and hierarchical characteristics of the crack patterns were accurately predicted. The numerical simulations allow a direct observation on whole the process of crack initiation and growth, which is quite a difficult task in experimental studies. The failure mechanisms and the fracture procedure are discussed according to the numerical results obtained from the simulations. It is shown that the numerical model is simple, robust, accurate and efficient in simulating crack evolution in real structures under thermal shock.
\end{abstract}

(C) 2013 Elsevier Ltd. All rights reserved.

Keywords: Thermal shock; Crack patterns; Non-local fracture model; Numerical simulations

\section{Introduction}

Ceramic materials are widely used in various industries due to their excellent high temperature mechanical properties, corrosion resistance, wear resistance, erosion resistance, oxidation resistance, etc. However, their inherent brittleness and insignificant ductility make them particularly vulnerable to thermal shock failure. In general, crack formation is considered as the major reason of failure in thermo-structural engineering. Understanding the mechanisms of cracking process in ceramics under thermal loads has been one of the most importance tasks in the research of this field.

Researches on fracture of ceramic materials underwent thermal shock was initiated 60 years ago by Kingery. ${ }^{1,2}$ He first proposed a so-called "critical stress" fracture criterion according to which cracks appear when the maximal thermal stress reaches the ultimate stress of the material. Hasselman proposed a different approach in which the driving force for crack propagation is

\footnotetext{
* Corresponding author. Tel.: +33 149402889; fax: +33 149403414 .

E-mail address: jia.li@univ-paris13.fr (J. Li).
}

derived from the elastic energy stored in the body at the instant of fracture. ${ }^{3}$

Afterward, numerous theoretical and experimental studies on thermal shock failure of ceramics have been reported. ${ }^{4-18}$ Among them, Hasselman gave qualitatively theoretical predictions of crack propagation behaviour in polycrystalline alumina rods under thermal shock by water quenching. ${ }^{5} \mathrm{Lu}$ and Fleck analyzed the thermal shock resistance of brittle solids by use of a stress-based fracture criterion. ${ }^{9}$ Bažant et al. and NematNasser et al. studied the stability of propagation of thermal shock cracks by using the energy principle, theoretically discussed the length hierarchy phenomenon of the crack patterns. ${ }^{12-14}$ This phenomenon was also studied theoretically and experimentally by Bahr et al. ${ }^{15-17}$ Jenkins has calculated the spacing and penetration of cracks by using a method based on energy minimization. ${ }^{18}$

Recently, Jiang et al. carried out experimental and numerical works in determining the crack patterns by taking the temperature-dependence of the material parameters into account. ${ }^{19}$ On the basis of a variational approach, Bourdin et al. developed a variational model capable to perform complicated fracture analysis in brittle materials under thermal shock. ${ }^{20}$ 
The crack pattern formation under thermal shock is quite a rapid and highly complex process. This process is difficult to capture with available experimental techniques. Only final crack patterns can easily be observed. This is why direct numerical simulations are particularly interesting in reproducing the cracking process. As a result, the failure mechanisms and the control parameters can be better understood. However, the direct numerical simulations have rarely been reported in the literature so far due to the inherent complexities in multi-cracking modelling. This is the main motivation of the present work.

In the previous works, the non-local and strain gradient schemes were used to model the damage and fracture in brittle materials. ${ }^{21-24}$ However, multi-cracking phenomenon such as that observed in thermal shock experiments is always an intrinsically difficult problem to deal with. In this paper, a non-local failure criterion was proposed and implemented into a finite element code. It was then applied to simulate the crack evolution in ceramic materials subjected to thermal shock. The proposed fracture model is equivalent to the maximum principal stress criterion for a specimen under pure tensile loading, and to the Griffith-Irwin criterion for crack propagation. Consequently, this non-local fracture model can both predict crack initiation as well as crack growth. The multi-cracking problems can easily be dealt with in a natural manner. Applying to the thermal shock problems, the proposed method successfully reproduces the crack patterns in ceramic specimens after quenching. The periodical and hierarchical characteristics of the crack patterns are predicted with satisfactory accuracy. Moreover, the direct numerical simulations faithfully describe whole the cracking process, including the crack initiation, crack growth and crack arrest during quenching tests. The numerical results are presented together with the previous experimental results. The comparison permits to evaluate the accuracy and efficiency of the proposed model. Finally, we give some concluding remarks and directions to follow in future works.

\section{Non-local damage model and numerical resolution method}

\subsection{Non-local fracture criterion}

We first outline the non-local fracture model used in this work. The basic idea of this model consists in replacing the local damage driving force, an effective stress $\sigma_{\mathrm{e}}$ for example, by its weighted average over a representative volume $V:{ }^{25}$

$\tilde{\sigma}_{\mathrm{e}}(x)=\frac{1}{\int_{V} \alpha(x) d V} \int_{V} \alpha(x-y) \sigma_{\mathrm{e}}(y) d y$

where $\alpha$ is a weighting function. In the present work, a coneshape function is adopted for simplicity:

$\alpha(r)=\left\{\begin{array}{cc}0 & r>R \\ 1-\frac{r}{R} & r \leq R\end{array}\right.$

where $r=\|x-y\| ; R$ is the radius of non-local action, representing a material characteristic length which defines the size of interaction zone in failure process.
We assume reasonably that the failure in ceramic materials under uniform stress fields obeys the maximum principal stress criterion. However, this criterion cannot directly be utilized to predict crack growth due to the stress singularity near the crack tips. To overcome this shorthand, we relate the maximum principal stress criterion to a crack growth criterion throughout a non-local formulation such like Eq. (1). Thus, the non-local maximum principal stress criterion can be written as follows:

$D= \begin{cases}0 & \tilde{\sigma}_{1}<\sigma_{c} \\ 1 & \tilde{\sigma}_{1} \geq \sigma_{c}\end{cases}$

where $D$ is the damage, $\sigma_{c}$ is the ultimate stress of the material, $\tilde{\sigma}_{1}$ is the non-local first principal stress. We enforce the validity of criterion (3) in two special cases: First, it should be valid in the case of a uniform tensile load. It is clear that in this case, $\tilde{\sigma}_{1}=\sigma_{1}$, and consequently, criterion (3) is equivalent to the maximum principal stress criterion. Second, it should be valid for the growth of a mode-I crack. To this end, we assume that the near-tip stress field is governed by the Williams asymptotic expansion. ${ }^{26}$ Therefore, for a mode-I loaded crack, the non-local first principal stress near the crack tip writes, according to (1) and (2):

$$
\begin{aligned}
\tilde{\sigma}_{1}(r, \theta)= & \frac{1}{\int_{0}^{R} \int_{-\pi}^{\pi}\left(1-\left(r^{\prime} / R\right)\right) r^{\prime} d r^{\prime} d \theta^{\prime}} \int_{0}^{R} \int_{-\pi}^{\pi} \\
& \times\left(1-\frac{r^{\prime}}{R}\right) \sigma_{1} r^{\prime} d r^{\prime} d \theta^{\prime}
\end{aligned}
$$

with $\sigma_{1}=\frac{K_{\mathrm{I}}}{\sqrt{2 \pi r}}\left(1+\left|\sin \frac{\theta}{2}\right|\right) \cos \frac{\theta}{2}$.

In these expressions, $K_{\mathrm{I}}$ is the stress intensity factor, $r$ and $\theta$ are the polar coordinates with the origin at the crack tip. Under mode I loading, the maximum non-local principal stress is located at a point on the crack axis near the crack tip $r=r_{0}, \theta=0$ due to the symmetry. We assume that $r_{0}$ is small such that the stress at its vicinity is still governed by the crack-tip asymptotic field.

On the one hand, according to the damage criterion (3), the element at $\left(r=r_{0}, \theta=0\right)$ is broken when $\tilde{\sigma}_{1} \geq \sigma_{c}$. On the other hand, from the Griffith-Irwin criterion of fracture, the crack grows when $K_{\mathrm{I}} \geq K_{\mathrm{Ic}}$, where $K_{\mathrm{Ic}}$ is the critical stress intensity factor. $^{27,28}$ This condition permits us to determine the non-local action radius $R$ and the location of the most loaded point $\left(r=r_{0}\right.$, $\theta=0$ ) by resolving numerically the following equation:

$$
\begin{aligned}
f(R)= & \sigma_{c}-\max _{r_{0}} \int_{0}^{R} \int_{-\pi}^{\pi} \frac{3}{\pi R^{2}}\left(1-\frac{r^{\prime}}{R}\right) \frac{K_{\mathrm{Ic}}}{\sqrt{2 \pi r}} \\
& \times\left(1+\left|\sin \frac{\theta}{2}\right|\right) \cos \frac{\theta}{2} r^{\prime} d r^{\prime} d \theta^{\prime}=0
\end{aligned}
$$

with

$$
r=\sqrt{\left(r_{0}+r^{\prime} \cos \theta^{\prime}\right)^{2}+\left(r^{\prime} \sin \theta^{\prime}\right)^{2}} \quad \tan \theta=\frac{r^{\prime} \sin \theta^{\prime}}{r_{0}+r^{\prime} \cos \theta^{\prime}}
$$

The geometrical quantities in Eq. (5) are shown in Fig. 1. Thus, the non-local damage criterion (3) is exactly equivalent to 


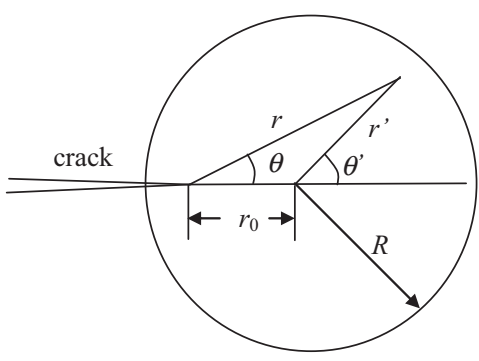

Fig. 1. Non-local action zone.

the Griffith-Irwin criterion when the non-local action radius $R$ is determined by (5). Consequently, we can confirm that in the cases of uniform tensile loads and mode-I cracks, the fracture can exactly be predicted by using the criterion (3). The other types of stress concentrations can be considered as intermediate states between these two special cases. In these cases, the fracture predicted by the present non-local model is a natural interpolation between these two extreme cases. From this point of view, the proposed non-local criterion can be used to predict the crack initiation as well as the crack propagation. In practice, we just need to find the point where the non-local principal stress is maximal: this point is broken when the non-local stress attaints the material strength.

\subsection{Numerical implementation}

The present non-local fracture criterion was implemented into a finite element code. In numerical simulations, we first evaluate the temperature evolution in the specimens. Then the thermal stresses are calculated by resolving the corresponding thermo-elasticity problem. The damage growth can therefore be assessed according to the criterion (3).

As the criterion (3) is an instantaneous damage model, an element is linearly elastic before its complete failure. Therefore, the crack propagation prediction is very similar to that adopted in the linear elastic fracture mechanics: a linear elastic calculation is first carried out for the cracked structure; then the crack propagation is determined according to a suitable criterion. This procedure is then repeated after each small crack progression in the structure. In the present work, the crack propagation is represented by successive eliminations of groups of damaged elements. Accordingly, the following algorithm is coded for thermal shock problems:

1. For a new time increment, calculate the temperature field;

2. Resolve the thermo-elasticity problem by using the finite element method;

3. Calculate the non-local effective stresses according to (1) and find the location $\mathbf{x}_{0}$ where the non-local effective stress is maximal;

4. Apply the non-local criterion (3): if $\tilde{\sigma}_{1}\left(x_{0}\right) \geq \sigma_{c}$, remove the elements within a small spot around $\mathbf{x}_{0}$; Go to step 2;

5. If $\tilde{\sigma}_{1}\left(x_{0}\right)<\sigma_{c}$, go to step 1 .

The radius of the damaged spots should be appropriately chosen in order to ensure that the broken zones form a

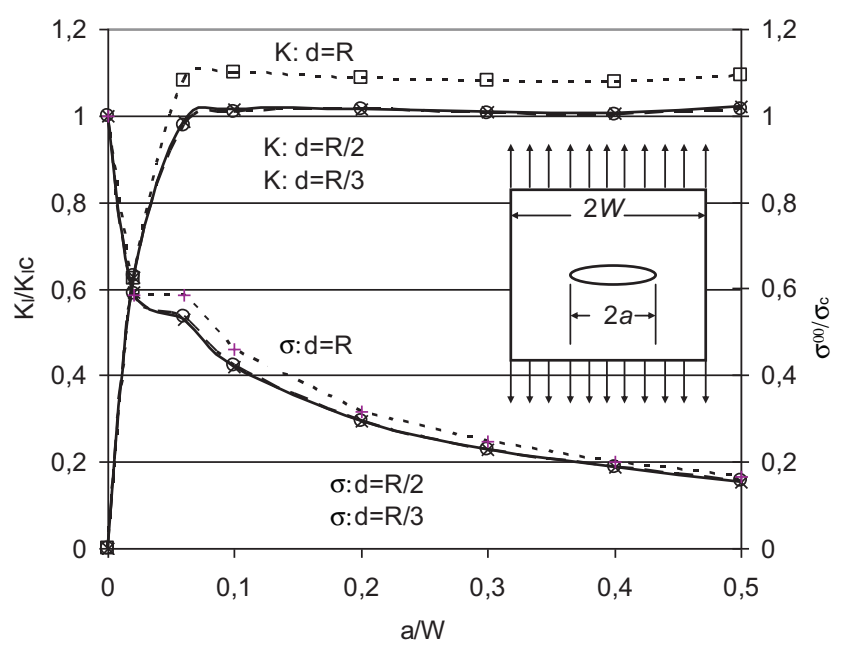

Fig. 2. Normalized stress intensity factor $K_{\mathrm{I}} / K_{\mathrm{Ic}}$ and normalized remote stresses $\sigma^{\infty} / \sigma_{c}$ as function of normalized semi-crack length $a / W$ for mode-I loaded cracks at fracture.

continuously growing crack: a too big spot will make the crack tip too blunted and will decrease the accuracy of the model in crack growth prediction. A too small value will considerably amplify the computational tasks.

\subsection{Validation of the numerical model}

In order to verify the accuracy of the method, we consider a plane stress plate containing a central crack. The mechanical properties of the material are: Young's modulus $E=370,000 \mathrm{MPa}$, Poisson's ratio $\nu=0.3$, the ultimate stress $\sigma_{c}=180 \mathrm{MPa}$, the critical stress intensity factor $K_{\mathrm{Ic}}=$ 94.87 $\mathrm{MPa} \sqrt{\mathrm{mm}}$. Thus the non-local action radius deduced from (5) is $R=0.1176 \mathrm{~mm}$ and the location of the maximal nonlocal first principal stress ahead of the crack tip is $r_{0} \approx 0.03 \mathrm{~mm}$. The plate was meshed with three-node plane stress elements. Different sizes of the elements near the crack tip are used in the simulations in order to investigate the influence of meshing, namely $d=R / 3, R / 2$ and $R$, where $d$ is the side length of the triangle elements. A pure tension along the normal direction of the crack was applied on the ends of the plate. By calculating the non-local first principal stresses from (1) and then finding their maximal value, we can easily determine the remote tensile stress at fracture according to the non-local fracture criterion (3). Then the stress intensity factor of such a central crack can be found from any handbook of stress intensity factors. ${ }^{29} \mathrm{We}$ compare the stress intensity factors thus obtained to the critical value $K_{\text {Ic }}$, and thus we can evaluate the accuracy of the method. The results of these calculations are shown in Fig. 2. In this figure, the stress intensity factors are normalized by the critical value $K_{\mathrm{Ic}}$; the remote stresses at fracture are normalized by the ultimate stress $\sigma_{c}$ of the material and the semi-crack length $a$ is normalized by the semi-plate width $W$.

From this figure, several remarks can be made:

1. When the crack is sufficiently long, the stress intensity factors at fracture evaluated from the present non-local 
damage criterion for crack growth equal correctly the critical stress intensity factor of the material. This result confirms that the proposed fracture model is equivalent to the Irwin criterion $K_{\mathrm{I}} \geq K_{\mathrm{Ic}}$ for a mode-I crack;

2. When the meshing is sufficiently fine, the proposed damage model is independent of the element size. Apart from the results calculated with $d=R$ that represents quite a coarse meshing, the calculations with finer meshes, e.g. $d=R / 3$ and $R / 2$, provide us with nearly identical remote loads at fracture. In fact, the non-local action zone should contain enough number of elements in order to guarantee a good accuracy on the calculation of non-local stresses. The element size $d=R / 2$ offers already sufficient accuracy for the crack growth prediction;

3. For short cracks, the present damage model is no longer equivalent to the Griffith criterion that is not suitable to predict the short crack growth. The critical stress intensity factor decreases as the crack length decreases. Moreover, the remote stress at fracture tends to the ultimate stress of the material as the crack length tends to zero. In this case, the proposed fracture criterion degenerates to the maximum principal stress criterion for non-singular stresses.

\section{Thermal shock problem}

In this section, we describe the application of the proposed non-local criterion in evaluating the cracking process in ceramic specimens under thermal shock.

\subsection{Experiments}

Jiang et al. carried out quenching experiments on ceramics plates from different temperatures. ${ }^{19}$ This experimental study will be used in the validation of the proposed model. In Jiang et al., $99 \% \mathrm{Al}_{2} \mathrm{O}_{3}$ powder was thermoformed into $50 \mathrm{~mm} \times 10 \mathrm{~mm} \times 1 \mathrm{~mm}$ thin specimens. They were bound up with inconel wires and heated to a temperature $T_{0}$ ranged from 300 to $600{ }^{\circ} \mathrm{C}$. After that, the heated specimens were dropped into a water bath of $T_{\infty}=20^{\circ} \mathrm{C}$ by free fall. Fig. 3 shows the thermal shock crack patterns. In order to remove the effect of the end boundaries, the regions within $10 \mathrm{~mm}$ from the two ends of the specimens were excluded in geometrical measures of the thermal shock cracks.

From Fig. 3, we can remark the following points:

- The number of cracks increases as the initial temperature increases;

- The lengths of the cracks increase as the initial temperature increases;

- The crack spacing decreases as the initial temperature increases;

- A tendency towards equal spacing between cracks can be observed;

- The crack patterns exhibit a hierarchical structure. One can distinguish 2 classes' cracks for quenching tests with

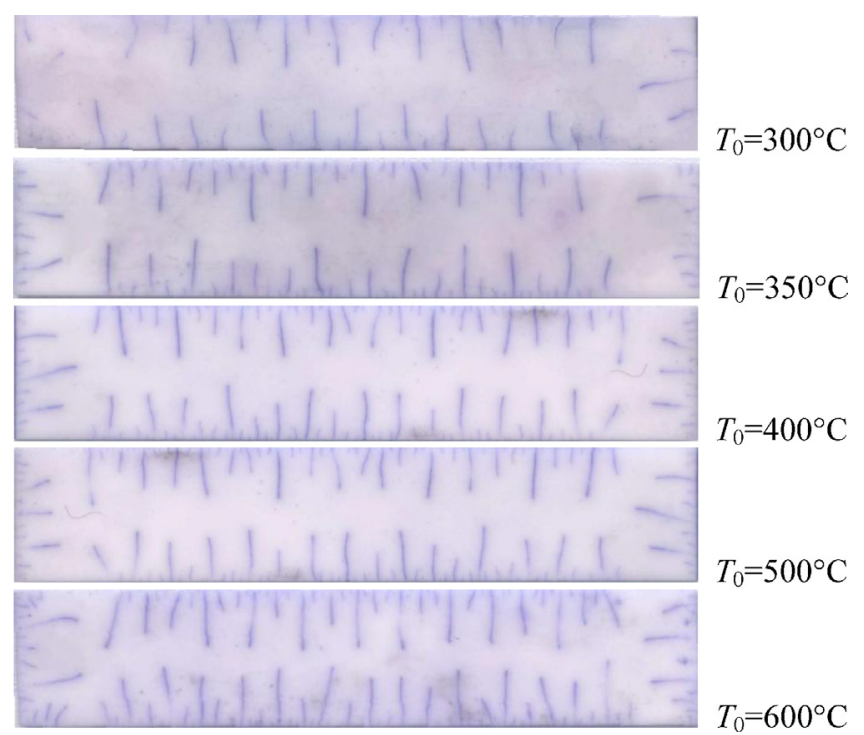

Fig. 3. Crack patterns after water quenching.

$T_{0}=300^{\circ} \mathrm{C}$, and 3 classes' cracks for quenching tests with $T_{0}=350-600^{\circ} \mathrm{C}$. However, the frontiers of the different classes are not always clear.

In the following, we will attempt to reproduce these properties of crack patterns by direct numerical simulations.

\subsection{Model}

By considering the symmetry of the specimen, only a quart of it was meshed by finite elements and modelled by using the proposed criterion. The specimen was meshed with threenode plane stress elements. The side size of the elements is about $d=0.05 \mathrm{~mm}$, e.g. about a half of the non-local action radius $R$. When the failure criterion is fulfilled in an element, it will be removed from the model, together with its immediate neighbouring elements. After elimination of the damaged elements, the new thermo-elasticity problem will be resolved on the basis of the changed mesh. As the distance between a crack tip and the position of the maximal non-local first principal stress is about $0.03 \mathrm{~mm}$ and the size of the damaged spot is enclosed approximately in a circle of $r=0.05-0.07 \mathrm{~mm}$, the successively removed spots must be overlapped and then connected. This technique ensures that the successive elimination of the damaged elements construct a continually growing macro-crack. As the damage bands formed from the successive damaged spots have a width of about $0.12 \mathrm{~mm}$, the damaged bands are not exactly sharp cracks. Numerical simulations show that the critical loads to propagate such damage bands are about 5\% higher than to propagate corresponding sharp cracks. Therefore, comparing to the specimen size $(50 \mathrm{~mm} \times 10 \mathrm{~mm})$, the damaged bands can still be regarded as sharp cracks without introducing notable errors. 


\subsection{Temperature fields}

The experimental conditions allow us to regard the temperature field as two-dimensional. Establish a Cartesian coordinate system Oxy with the origin at the centre of the specimen and $x$ and $y$ coinciding with the specimen axes. Noting that during the process of water quenching, the water temperature holds at $T_{\infty}=20^{\circ} \mathrm{C}$ in the bath. The heat-conduction equation writes:

$a\left(\frac{\partial^{2} T}{\partial x^{2}}+\frac{\partial^{2} T}{\partial y^{2}}\right)=\frac{\partial T}{\partial t}$

where $t$ is the time, $a=k / \rho c ; k, \rho, c$ are the thermal conductivity, density and specific heat, respectively. The initial temperature is assumed to be uniform in the specimen:

$T(x, y, t=0)=T_{0}$

where $T_{0}$ is the preset temperature before quenching and its values are $300{ }^{\circ} \mathrm{C}, 350{ }^{\circ} \mathrm{C}, 400{ }^{\circ} \mathrm{C}, 500^{\circ} \mathrm{C}$ and $600{ }^{\circ} \mathrm{C}$, respectively. Noting that the $x$-axis and $y$-axis are the symmetrical axes of temperature, the boundary conditions of the model are therefore

$$
\begin{aligned}
& \frac{\partial T}{\partial y}=0 \quad \text { on the } x \text {-axis } \\
& \frac{\partial T}{\partial x}=0 \quad \text { on the } y \text {-axis }
\end{aligned}
$$$$
-k \frac{\partial T}{\partial x}=h\left[T-T_{\infty}\right] \quad \text { on the left boundary }
$$$$
-k \frac{\partial T}{\partial y}=h\left[T-T_{\infty}\right] \quad \text { on the bottom boundary }
$$

where $h$ is the convective heat transfer coefficient.

The transient temperature field $T=T(x, y, t)$ in the specimen can be expressed as follows:

$$
\begin{aligned}
\frac{T}{T_{0}}= & {\left[\sum_{m=1}^{\infty} X_{m} \exp \left(-\alpha_{m}^{2} \frac{a t}{L_{1}^{2}}\right) \cos \left(\alpha_{m} \frac{x}{L_{1}}\right)\right] } \\
& \times\left[\sum_{m=1}^{\infty} Y_{m} \exp \left(-\beta_{m}^{2} \frac{a t}{L_{2}^{2}}\right) \cos \left(\beta_{m} \frac{y}{L_{2}}\right)\right]
\end{aligned}
$$

where

$$
\begin{aligned}
X_{m} & =\frac{2 \sin \alpha_{m}}{\alpha_{m}+\sin \alpha_{m} \cos \alpha_{m}} \\
Y_{m} & =\frac{2 \sin \beta_{m}}{\beta_{m}+\sin \beta_{m} \cos \beta_{m}}
\end{aligned}
$$

with $\alpha_{m}$ and $\beta_{m}$ are the roots of the following transcendent equations:

$$
\begin{aligned}
\tan \alpha_{m} & =\frac{h L_{1}}{k \alpha_{m}} \\
\tan \beta_{m} & =\frac{h L_{2}}{k \beta_{m}}
\end{aligned}
$$

where $L_{1}$ and $L_{2}$ are respectively the semi-length and semiwidth of the specimen.

After numerical resolution of (10), the temperature field can directly be obtained from (9).
Table 1

Mechanical and thermal parameters used in the simulations.

\begin{tabular}{llll}
\hline$E(\mathrm{MPa})$ & $v$ & $\sigma_{c}(\mathrm{MPa})$ & $\rho\left(\mathrm{kg} / \mathrm{m}^{3}\right)$ \\
\hline 370,000 & 0.3 & 180 & 3980 \\
$G\left(\mathrm{~J} / \mathrm{m}^{2}\right)$ & $k(\mathrm{~W} /(\mathrm{m} \mathrm{K}))$ & $c(\mathrm{~J} /(\mathrm{kg} \mathrm{K}))$ & $\alpha$ \\
\hline 24.3 & 31 & 880 & $7.5 \times 10^{-6}$ \\
\hline
\end{tabular}

An important hypothesis adopted here is that the cracking process will not influence on the thermal transfer regime. This hypothesis simplifies the temperature calculations during the cracking process.

\subsection{Material parameters}

The ceramic specimens were assumed to be linearly elastic, homogeneous and isotropic. The mechanical and thermal characteristics are assumed to be constant and not influenced by temperature variation. This assumption is quite correct for mechanical parameters. From available data, Young's modulus $E$, Poisson's ratio $\nu$, the ultimate tensile stress $\sigma_{c}$, the density $\rho$, and the energy release rate $G$ of $99 \% \mathrm{Al}_{2} \mathrm{O}_{3}$ ceramics are listed in Table $1 .{ }^{30,31}$ The critical stress intensity factor is deduced from $K_{\mathrm{Ic}}=\sqrt{G E}$. The non-local action radius deduced from (5) is $R=0.1176 \mathrm{~mm}$.

Conversely, the thermal parameters such like the thermal conductivity $k$, the specific heat $c$ and the thermal expansion coefficient $\alpha$ are temperature-dependent. ${ }^{32-34}$ Consequently, adopting constant values in the quenching simulations is an approximate and simplified assumption. The values used in the simulations are listed in Table $1 .^{32-34}$

Precaution in numerical simulations should be made on the choice of the convective heat transfer coefficient $h$ in thermal shock. The available data give scattering estimations of this parameter and the mutual deviation is high up to an order of magnitude $\left(h \approx 10^{4}-10^{5} \mathrm{~W} /\left(\mathrm{m}^{2} \mathrm{~K}\right)\right) .{ }^{35-39}$ In this work, a constant value for $h$ was used in all the simulations, its value was estimated such that the numerical results on average crack spacing at each quenching temperature approximately agree with the experimental measurement. The value used in the present work is $h=50,000 \mathrm{~W} /\left(\mathrm{m}^{2} \mathrm{~K}\right)$.

\section{Results and discussions}

\subsection{Direct comparison with the experiments}

Fig. 4 illustrates the final crack patterns of the simulations for different initial quenching temperature. For comparison, the experimentally obtained crack patterns are also showed. From these figures, we can make a direct judgement on the quality of the numerical simulations. Globally speaking, the simulations reproduce faithfully the crack patterns obtained from quenching tests. The resemblance between the numerical results and the real tests is obvious. Following remarks can be made: 

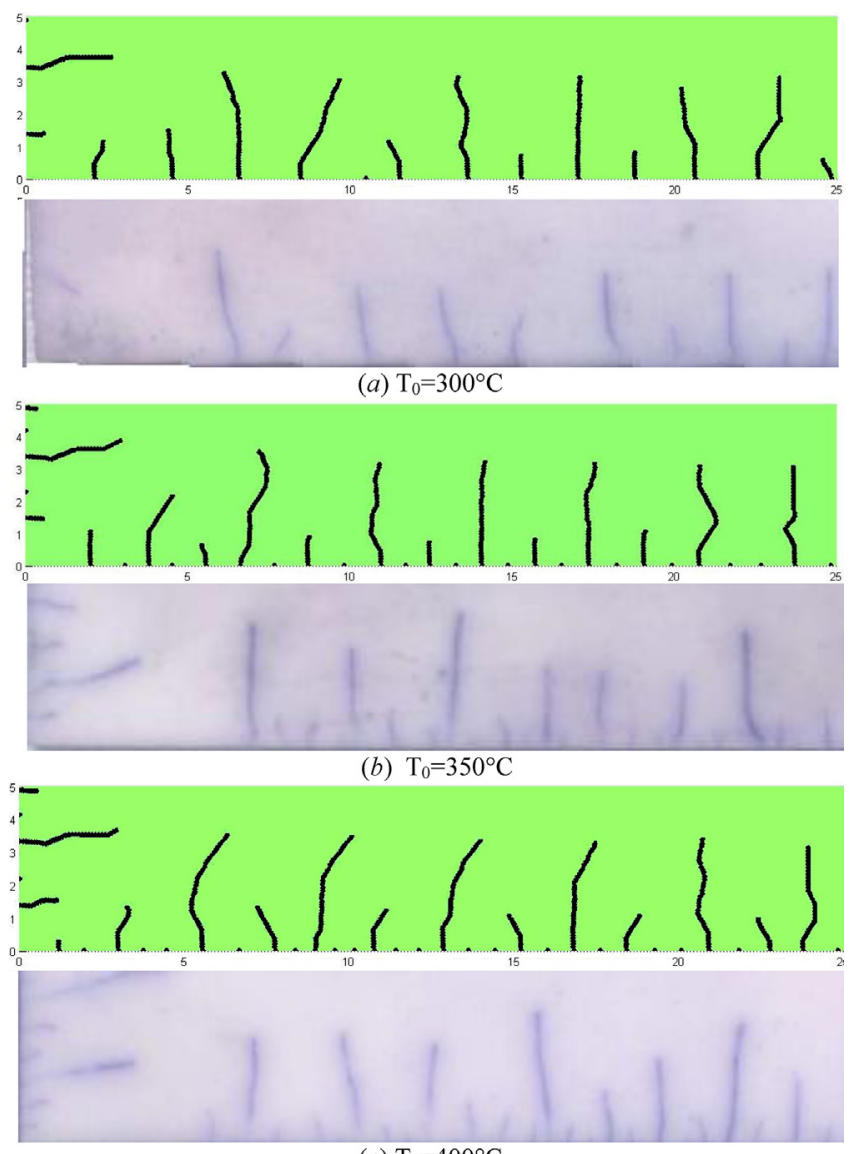

(c) $\mathrm{T}_{0}=400^{\circ} \mathrm{C}$

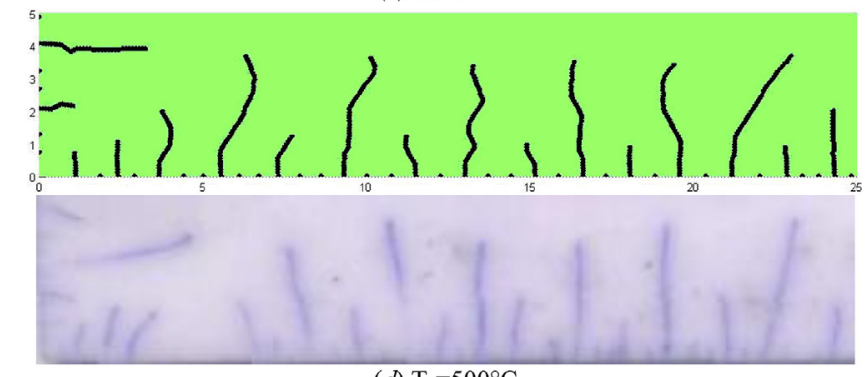

(d) $\mathrm{T}_{0}=500^{\circ} \mathrm{C}$

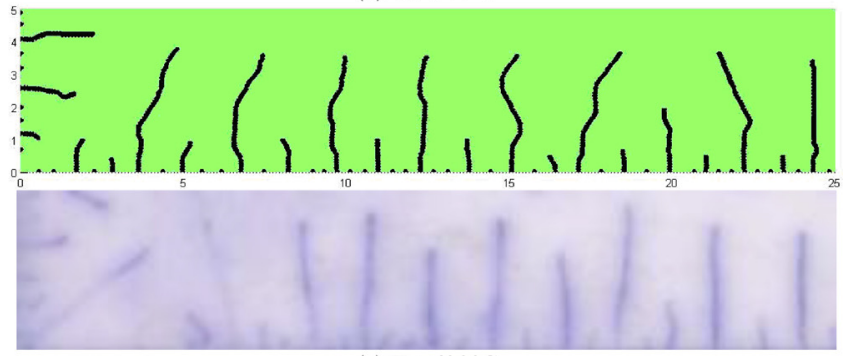

(e) $\mathrm{T}_{0}=600^{\circ} \mathrm{C}$

Fig. 4. Comparison between the crack patterns in numerical models and in real specimens.

1. As in quenching tests, multi-cracking patterns are obtained. The cracks initiate on all the contour and propagate into the interior of the specimens;

2. As in quenching tests, the cracks can be classified into several hierarchical levels. For $T_{0}=300^{\circ} \mathrm{C}$, two hierarchical classes of cracks can be observed, while for $T_{0}=350^{\circ} \mathrm{C}$, $400^{\circ} \mathrm{C}, 500^{\circ} \mathrm{C}$ and $600^{\circ} \mathrm{C}$, we can find three classes of cracks.

3. A tendency towards equal spacing between cracks is obvious in both the numerical and real crack patterns.

\subsection{Characteristic values of the crack patterns}

The numerical simulations reproduce the periodic and hierarchical crack patterns appeared in the quenching tests. In the present study, the numerical simulations provide more regular crack patterns comparing to those in real specimens. This is because that the material heterogeneities in the real material at microscopic level have not been included in the modelling. However, statistically speaking, the principal features of the crack patterns can be brought out from numerical results. Table 2 shows the comparison between the experimental and numerical results on average crack spacing $s$ observed at the specimen surfaces. From this comparison, we can see the numerical simulations are quite accurate, even by using constant material parameters.

Although the morphologies are very similar between the experimental crack patterns and the numerical ones, the quantitative comparison on crack lengths $p$ is not evident due to the data scattering of the experimental results. By assuming that each crack level obeys a Gaussian distribution, Jiang et al. described the crack length distribution by a combined Gaussian function. ${ }^{19}$ This analysis allowed the classification of the cracks according to their length in a statistical manner. In Table 3, we list the average lengths of the longest cracks of the experimental and numerical crack patterns. The comparison shows a good agreement, even though the numerical simulations give smaller crack lengths comparing to the experiments.

The principal reason of this difference may be the blunt form of the crack tips in numerical models, in which the cracks were represented by damaged bands with a finite thickness.

Hereafter we give a global comparison on crack length distribution between the experiments and simulations. Fig. 5 shows the histograms of the numerical results and the curves of the experimental results on frequency distribution of dimensionless crack length $\bar{p}=p / L_{2}$ for all the quenching temperatures. In measuring the geometry of the thermal shock cracks, to remove the effects of the end boundaries, the regions within $10 \mathrm{~mm}$ from the two ends of the specimens were excluded. It is clear that this comparison is not perfectly significant as the simulations were carried out with one specimen quarter, while the experiment data were obtained from statistics over five specimens for each temperature. Consequently, the random effects existed in the tests are not taken into account in numerical simulations. Nevertheless, this comparison shows an overall agreement between the numerical and experimental results. Globally speaking, the total numbers of cracks, therefore the average crack spacing, observed in the tests and simulations are quite close, while the different classes of cracks obtained in the simulations are somewhat shorter than those observed in the tests. This difference may be caused by the blunt form of the crack tips in numerical models. These results confirm those shown in Tables 2 and 3. 
Table 2

Dimensionless average crack spacing $\bar{s}=s / L_{2}$.

\begin{tabular}{|c|c|c|c|c|c|c|c|c|c|c|}
\hline \multirow[t]{2}{*}{$T_{0}$} & \multicolumn{2}{|c|}{$300^{\circ} \mathrm{C}$} & \multicolumn{2}{|c|}{$350^{\circ} \mathrm{C}$} & \multicolumn{2}{|c|}{$400^{\circ} \mathrm{C}$} & \multicolumn{2}{|c|}{$500^{\circ} \mathrm{C}$} & \multicolumn{2}{|c|}{$600^{\circ} \mathrm{C}$} \\
\hline & Test & Simulation & Test & Simulation & Test & Simulation & Test & Simulation & Test & Simulation \\
\hline $\bar{s}$ & 0.32 & 0.333 & 0.184 & 0.181 & 0.16 & 0.158 & 0.142 & 0.133 & 0.12 & 0.105 \\
\hline
\end{tabular}

Table 3

Dimensionless crack length $\bar{p}=p / L_{2}$.

\begin{tabular}{|c|c|c|c|c|c|c|c|c|c|c|}
\hline \multirow[t]{2}{*}{$T_{0}$} & \multicolumn{2}{|c|}{$300^{\circ} \mathrm{C}$} & \multicolumn{2}{|c|}{$350^{\circ} \mathrm{C}$} & \multicolumn{2}{|c|}{$400^{\circ} \mathrm{C}$} & \multicolumn{2}{|c|}{$500^{\circ} \mathrm{C}$} & \multicolumn{2}{|c|}{$600^{\circ} \mathrm{C}$} \\
\hline & Test & Simulation & Test & Simulation & Test & Simulation & Test & Simulation & Test & Simulation \\
\hline $\bar{p}$ & 0.72 & 0.64 & 0.74 & 0.65 & 0.72 & 0.68 & 0.78 & 0.73 & 0.8 & 0.75 \\
\hline
\end{tabular}

\subsection{Diagrams on crack spacing against crack length}

Bahr et al. argued that the diagram crack spacing vs crack length can serve as justification for applying a model to a real material. ${ }^{17}$ Fig. 6 shows the comparison between the experimental and numerical results on such curves. The average crack spacing $s$ has been determined by counting the intersection points of cracks with a straight line in the depth $p$. These values were normalized by the semi-width of the specimens to obtained the corresponding dimensionless values $\bar{s}=s / L_{2}$ and $\bar{p}=$ $p / L_{2}$. It is shown that the numerical model presents satisfactory agreement with the real experiments despite of randomness involved in the latter. The absence of the distinct hierarchy of crack lengths in real specimens is apparently due to the randomness of initial flaws and non-determinist aspects in experiments. In contrast, the numerical results exhibit several jumps and plateaux. In view of the fact that no parameter fitting is involved in the data of Fig. 4, model and experiment agree unexpectedly well.

\subsection{Cracking process during the thermal shock}

In general, it is very difficult to observe the crack initiation and crack growth during water quenching by means of experimental techniques. Therefore, the numerical description on cracking process is the unique method to this end so far. Moreover, it can provide us with supplementary information on understanding the fracture mechanisms. Fig. 7 shows the first crack initiations at the specimen surface. The distribution of the first principal stresses is also shown by means of a colouring map. From this image, we can observe that, at a critical time where the temperature gradient reaches a sufficiently high level, the first crack will appear at the location where the non-local first principal stress is maximal and fulfils the failure criterion (3). After that, the stresses are relaxed at this location, and the stress redistribution gives another location where the first principal stress is maximal. If the fracture criterion (3) is always fulfilled, a new crack onset takes place. Since the stress distribution is nearly uniform along the specimen surface, the first crack initiations are somewhat randomly located, depending only on the scattering of the numerical results. The following cracks will appear between the most distant cracks previously formed. This procedure will repeat until the temperature gradient is no longer capable to produce sufficient stress concentration to onset new cracks.

Fig. 8 shows some different steps of the cracking process. The crack growth scheme can be summarized by observing these images. At the beginning, the thermal shock cracks initiate and propagate uniformly with a nearly equal spacing (Fig. 8a). At this step, the thermal shock cracks propagate simultaneously and very rapidly, then the propagation speed decreases gradually with release of the thermal stresses until the strain energy cannot support simultaneous propagation of all the cracks. Consequently, only a reduced number of cracks continue to propagate, whereas the other cracks stop. The crack spacing increases until 2 or 3 times larger than the previous one (Fig. 8b). In the following steps, the crack growth may deviate and attempt to form equal crack spacing (Fig. 8c). This procedure may repeat several times until the strain energy induced by thermal stresses cannot support propagation of any crack. The final simulation crack patterns (Fig. 8d) are very similar to those observed in experimental results. This remark supports the cracking process above described.

The colouring map represents the levels of first principal stresses (unity in MPa). From Fig. 8, the locations of stress concentrations can clearly be observed. The competition amongst the stress concentrations at different locations leads to the formation of multi-crack failure patterns. The proposed damage model provides a natural manner in reproducing them. Comparing to the traditional treatment of fracture mechanics in which the crack growth conditions should be examined at each crack tip, the present method is much more simple and robust to deal with multi-cracking problems.

\section{Conclusions and future works}

In this work, a non-local failure model was described to predict crack initiation and crack growth in brittle materials and then implemented into a finite element code. This model is equivalent to the maximum principal stress criterion for a specimen under pure tensile loading, and to the Griffith-Irwin criterion for the crack propagation. This non-local fracture model was successfully applied to simulating the crack evolution in ceramic materials subjected to thermal shock. From the results of the 


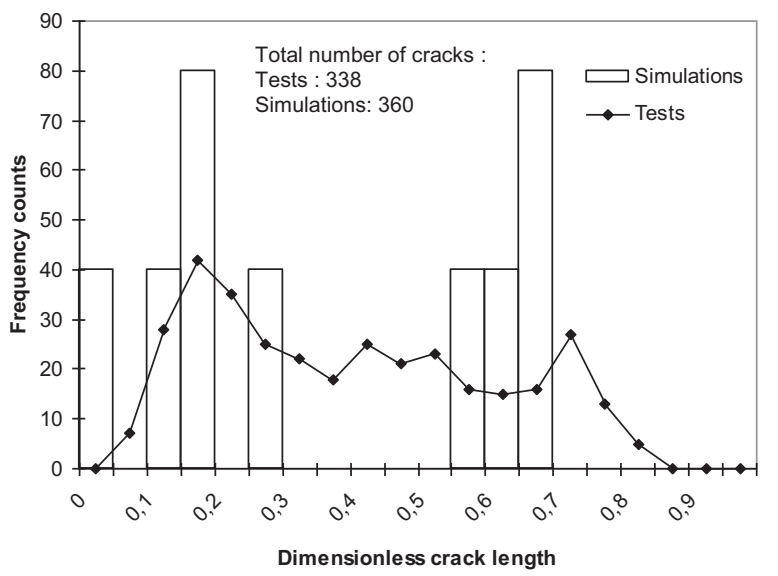

(a) $\mathrm{T}_{0}=300^{\circ} \mathrm{C}$

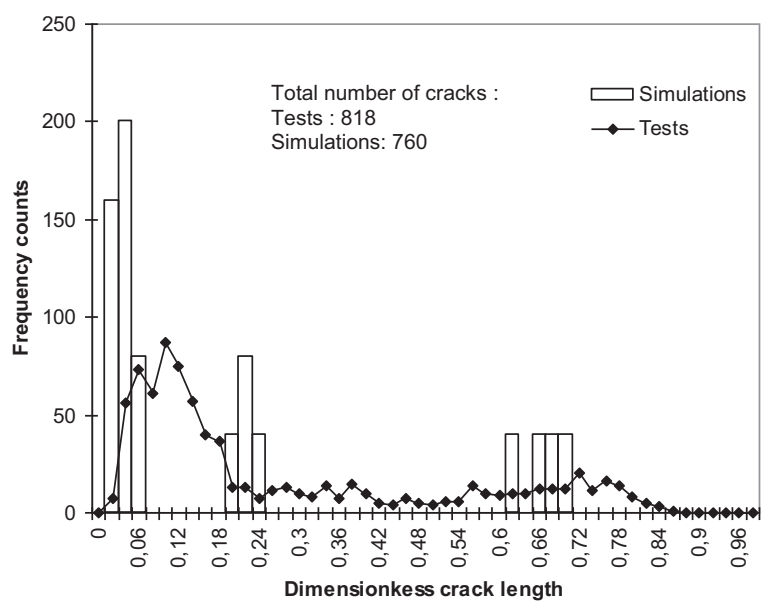

(c) $\mathrm{T}_{0}=400^{\circ} \mathrm{C}$

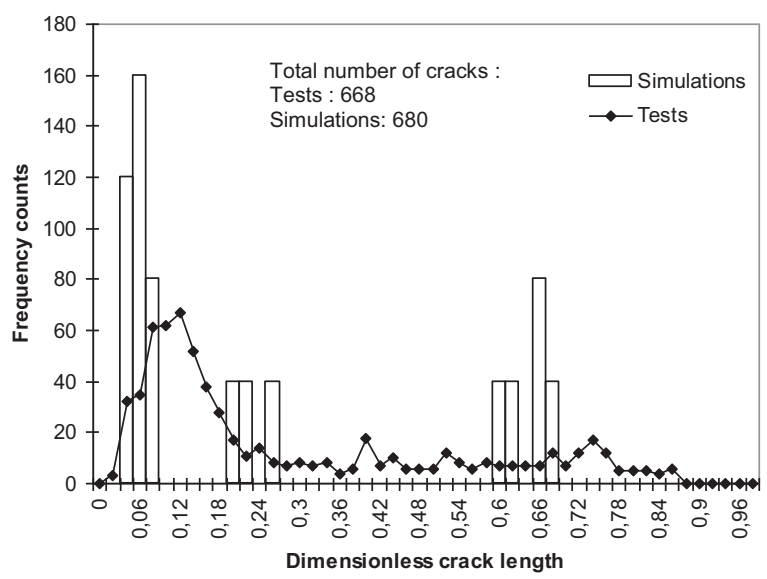

(b) $\mathrm{T}_{0}=350^{\circ} \mathrm{C}$

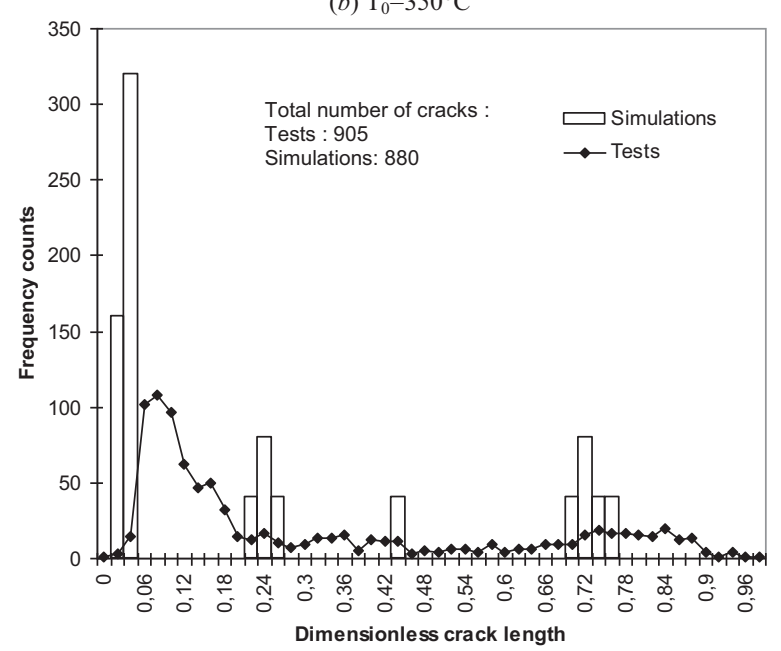

(d) $\mathrm{T}_{0}=500^{\circ} \mathrm{C}$

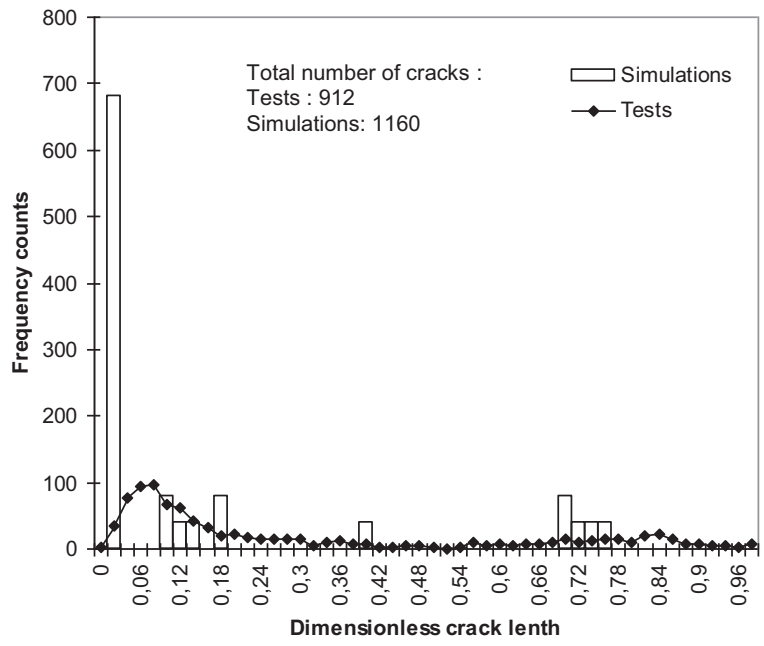

(e) $\mathrm{T}_{0}=600^{\circ} \mathrm{C}$

Fig. 5. Frequency distribution of dimensionless crack length.

numerical simulations, the following conclusions can be formulated:

1. The numerical simulations reproduced faithfully the crack patterns in ceramic specimens after quenching tests. The periodical and hierarchical characteristics of the crack patterns were accurately predicted;

2. The parameters describing the crack patterns such as the average crack spacing and the crack lengths were correctly estimated from the numerical results; 

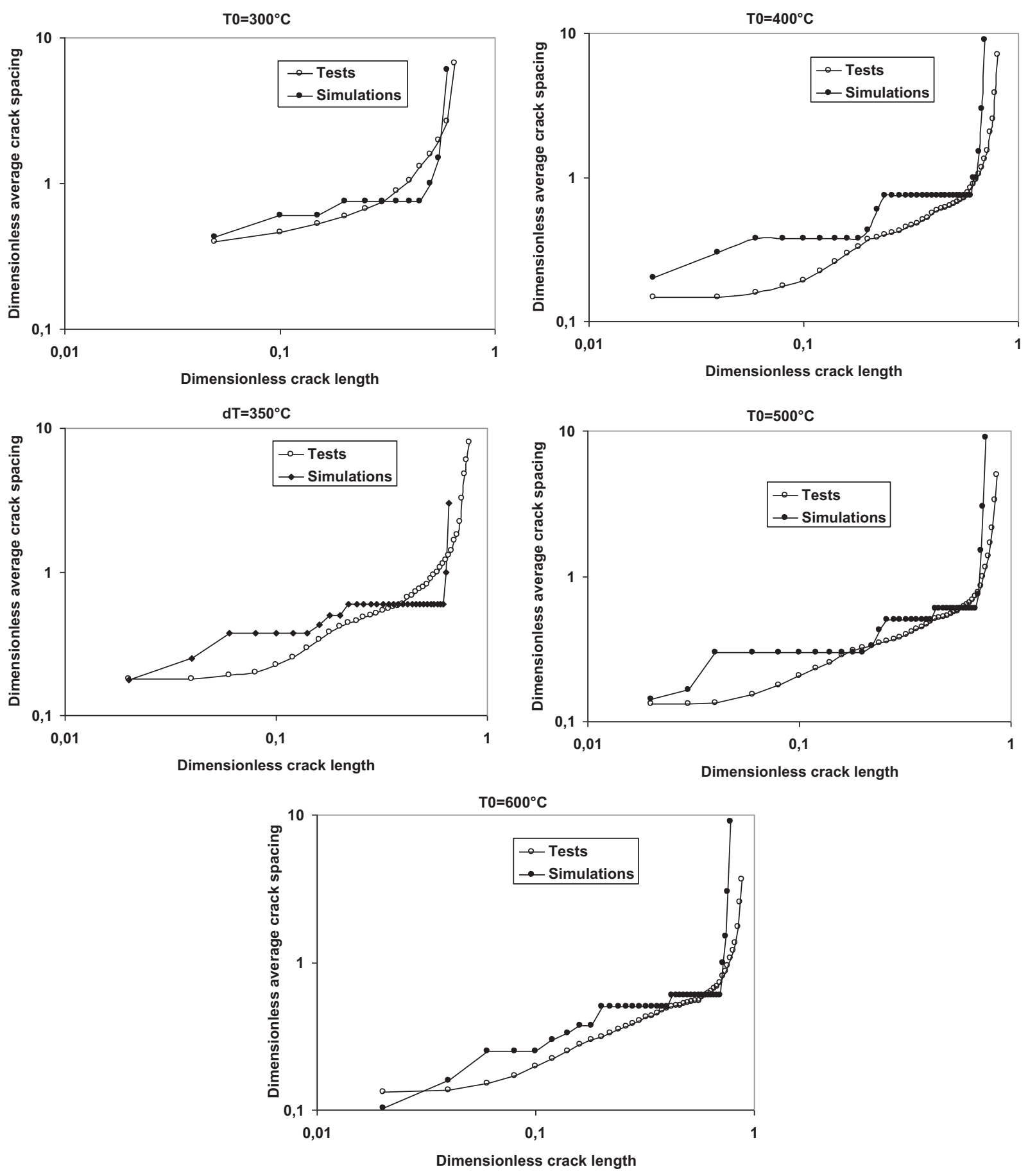

Fig. 6. Crack spacing vs crack length.

3. The numerical simulations allow a direct observation on crack initiation and growth in the specimens, which is quite a difficult task in experimental studies.

4. The proposed non-local damage model is based on reel material characteristics habitually used in engineering applications. No parameter fitting is required to improve the quality of the proposed model. The finite element implementation of the present non-local criterion allows accurate cracking simulations for real structures under thermal shock. The theoretical concept is clear and simple. The numerical model is robust, easy to apply to different engineering structures subjected to thermal shock.

It is clear that further development is also necessary to improve the accuracy and efficiency of the proposed model and to extend it to more general cases. For examples, consideration of the temperature-dependency of the thermal parameters; consideration of dynamics effect; extension to 3D structures etc. could be important issues of the future works. 


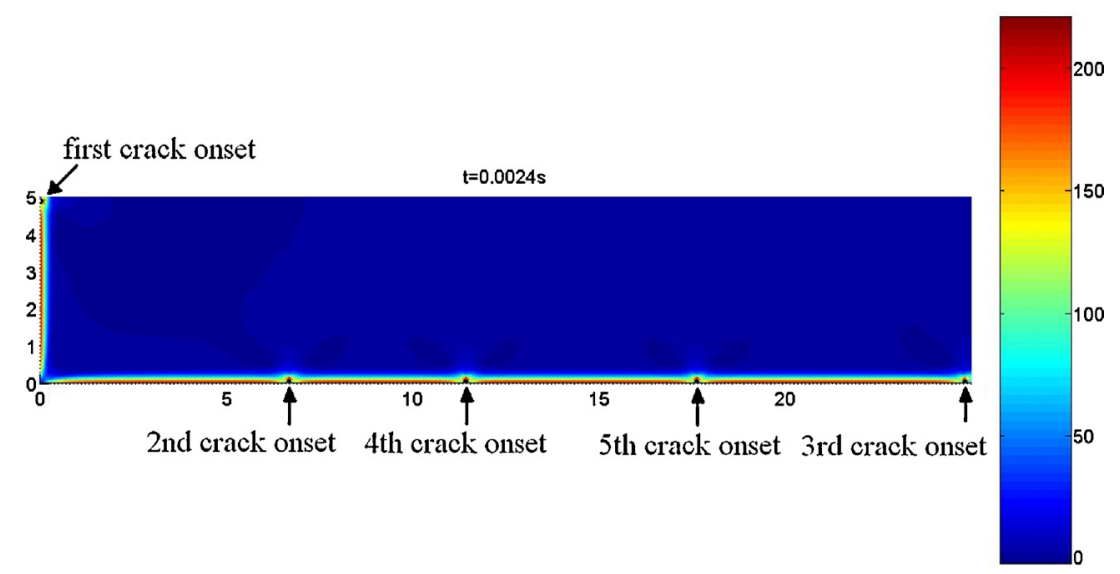

Fig. 7. First crack initiations.

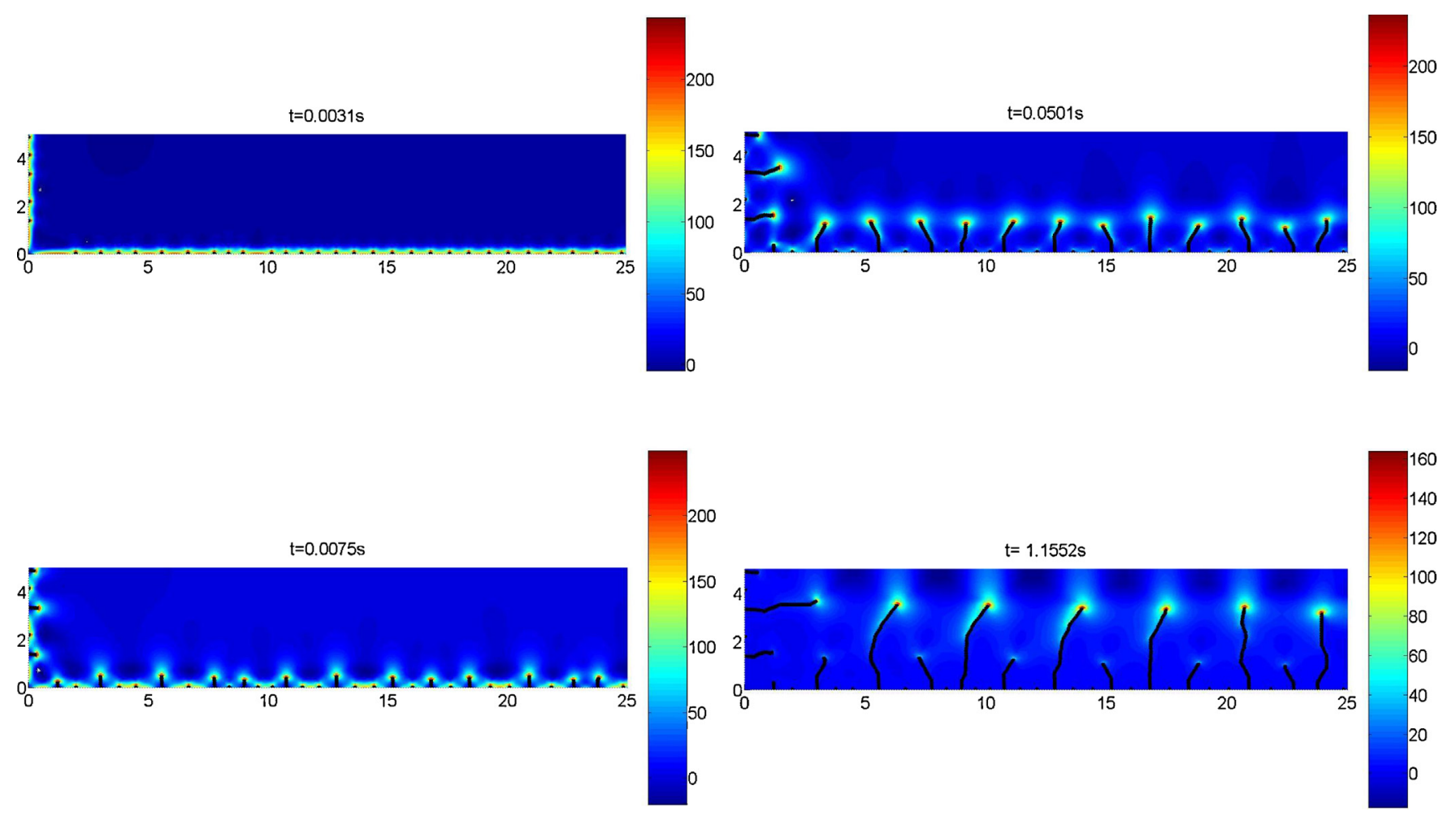

Fig. 8. Crack patterns at different times, $T_{0}=500^{\circ} \mathrm{C}$.

\section{Acknowledgements}

This work was supported by the funding from the French ANR program T-Shock ANR-10-INTB-0915 and the National Natural Science Foundations of China (Grants Nos. 11061130550 and 11172023).

\section{References}

1. Kingery WD. Metal-ceramic interaction: IV, absolute measurement of metal-ceramic interfacial energy and interfacial adsorption of silicon from iron-silicon alloys. J Am Ceram Soc 1954;37:42-5.

2. Kingery WD. Factors affecting thermal stress resistance of ceramic materials. J Am Ceram Soc 1955;38:3-15.
3. Hasselman DPH. Approximate theory of thermal stress resistance of brittle ceramics involving creep. J Am Ceram Soc 1969;50:454-7.

4. Mai YW. Thermal stress resistance and fracture toughness of two tools ceramics. J Mater Sci 1976;11:1430-8.

5. Hasselman DPH. Strength behaviour of polycrystalline alumina subjected to thermal shock. J Am Ceram Soc 1970;53(9):490-5.

6. Collin M, Rowcliffe D. Analysis and prediction of thermal shock in brittle materials. Acta Mater 2000;48(8):1655-65.

7. Gupta TK. Strength degradation and crack propagation in thermally shocked alumina. J Am Ceram Soc 1972;55(5):249-53.

8. Coppola JA, Hasslman DPH. Strength loss of brittle ceramics subjected to severe thermal shock. J Am Ceram Soc 1972;55(9):481-9.

9. Lu TJ, Fleck NA. The thermal shock resistance of solids. Acta Mater 1998;46(13):4755-68.

10. Holmquist M, Lundberg R, Sudre O, Razzell AG, Molliex L, Benoit J, et al. Alumina/alumina composite with a porous zireonia interphase-processing, properties and component testing. J Eur Ceram Soc 2000;20:599-606. 
11. Wang L, Shi JL, Gao JH, Yan DS. Influence of tungsten carbide particles on resistance of alumina matrix ceramics to thermal shock. J Eur Ceram Soc 2001;21:1213-7.

12. Bažant ZP, Ohtsubo H, Aoh K. Stability and post-critical growth of a system of cooling or shrinkage cracks. Int J Fract 1979;15(5):443-56.

13. Nemat-Nasser S. Stability of a system of interacting cracks. Int J Eng Sci 1978;16(4):277-85.

14. Nemat-Nasser S, Keer LM, Parihar KS. Unstable growth of thermally induced interacting cracks in brittle solids. Int J Solids Structures 1978;14(6):409-30.

15. Bahr HA, Fischer G, Weiss HJ. Thermal-shock crack patterns explained by single and multiple crack propagation. J Mater Sci 1986;21(8):2716-20.

16. Bahr HA, Balke H, Kuna M, Liesk H. Fracture analysis of a single edge cracked strip under thermal shock. Theor Appl Fract Mech 1987;8(1):33-9.

17. Bahr HA, Weiss HJ, Maschke HG, Meissner F. Multiple crack propagation in a strip caused by thermal shock. J Mech Phys Solids 2010;58:1411-21.

18. Jenkins DR. Optimal spacing and penetration of cracks in a shrinking slab. Phys Rev E 2005;71(5):056117.

19. Jiang CP, Wu XF, Li J, Song F, Shao YF, Xu XH, et al. A study of the mechanism of formation and numerical simulations of crack patterns in ceramics subjected to thermal shock. Acta Mater 2012;60:4540-50.

20. Bourdin B, Maurini C. A variational approach to thermal fracture. In: XXIII ICTAM. 2012

21. Li J. A micromechanics-based strain gradient damage model for fracture prediction of brittle materials_-part I: homogenization methodology and constitutive relations. Int J Struct Solids 2011;48:3336-45.

22. Li J, Pham T, Abdelmoula R, Song F, Jiang CP. A micromechanicsbased strain gradient damage model for fracture prediction of brittle materials_-part II: damage modeling and numerical simulations. Int J Struct Solids 2011;48:3346-58.

23. Li J, Tian XX, Abdelmoula R. A damage model for crack prediction in brittle and quasi-brittle materials solved by the FFT method. Int $J$ Fract 2012;173:135-46.

24. Li J, Meng SH, Tian XX, Song F, Jiang CP. A non-local fracture model for composite laminates and numerical simulations by the FFT method. Composites B 2012;43:961-71.
25. Pijaudier-Cabot G, Bazant ZP. Nonlocal damage theory. J Eng Mech ASCE 1987;113:1512-33.

26. Williams ML. Stress singularities resulting from various boundary conditions in angular corners of plates in extension. ASME J Appl Mech 1952;19:526-8.

27. Griffith AA. The phenomena of rupture and flow in solids. Phil Trans $R$ Soc Lond 1920;221:163-98.

28. Irwin G. Linear fracture mechanics, fracture transition and fracture control. Eng Fract Mech 1968;1:241-57.

29. Sih G. Handbook of stress-intensity factors. Lehigh University; 1973.

30. Zhang YL, Ma JP. Applicable ceramic material manual. Beijing: Chemical Industry Press; 2006 (in Chinese).

31. Zhang QC. Mechanical properties of ceramics. Beijing: Science Press; 1987 (in Chinese).

32. Touloukian YS, Ho CY. Thermophysical properties of matter thermal conductivity of nonmetallic solids, vol. 2. New York: Plenum Press; 1972

33. Touloukian YS, Ho CY. Thermophysical properties of matter specific heat of nonmetallic solids, vol. 5. New York: Plenum Press; 1972.

34. Jiang DL, Li LT, Ouyang SHX, Shi JL. China materials engineering canon. Beijing: Chemical Industry Press; 2006 (in Chinese).

35. Singh JP, Tree Y, Hasselman DPH. Effect of bath and specimen temperature on the thermal stress resistance of brittle ceramics subjected to thermal quenching. J Mater Sci 1981;16(8):2109-18.

36. Becher PF. Effect of water bath temperature on the thermal shock of $\mathrm{Al}_{2} \mathrm{O}_{3}$. J Am Ceram Soc 1981;64(1):C17-8.

37. Kim Y, Lee WJ, Case ED. The measurement of the surface heat transfer coefficient for ceramics quenched into a water bath. Mater Sci Eng A 1991;145(1):L7-11.

38. Lee WJ, Kim Y, Case ED. The effect of quenching media on the heat transfer coefficient of polycrystalline alumina. J Mater Sci 1993;28(8): 2079-83.

39. Zhou ZL, Song F, Shao YF, Meng SH, Jiang CP, Li J. Characteristics of the surface heat transfer coefficient for $\mathrm{Al}_{2} \mathrm{O}_{3}$ ceramic in water quench. $J$ Eur Ceram Soc 2012;32:3029-34. 